\title{
Prognosis of patients with acute renal failure without cardiopathy
}

\author{
N Gallego, C Pérez-Caballero, A Gallego, R Estepa, F Liaño, J Ortuño
}

\begin{abstract}
Background-The outcome for children with acute renal failure (ARF) may be poor. However, relatively few published studies have considered prognosis of these patients.

Methods-We prospectively studied, from 1978 to 1998,92 such children without heart disease to try to identify risk factors for mortality.

Results-Forty five per cent of children with tumours, shock, and other causes died compared with none of those with a primary urinary tract related problem. ARF did not seem to be the cause of death in any case. Univariate analysis showed that in the non-primary urinary problem group (55 cases), patients with hypotension, high values of BUN or creatinine, or who needed mechanical ventilation or dialysis, had a poor outcome. Multivariate analysis showed that probability of death can be estimated using the following score: $-0.02+0.28$ (hypotension) +0.19 (ventilation) +0.27 (dialysis) +0.01 (BUN).

Conclusions-Mortality of patients with ARF was related to aetiology, the need for dialysis and/or ventilator use, hypotension, and BUN values.

(Arch Dis Child 2001;84:258-260)
\end{abstract}

Keywords: acute renal failure; multivariate analysis;

Acute renal failure (ARF) in children has a poor prognosis, despite the development of modern techniques for treating the syndrome. ${ }^{1}$ Children may develop ARF which is associated with substantial mortality during the course of extrarenal disease. ${ }^{2}$

Although a great deal of work about this

Servicio de Nefrología, Hospital Ramón y Cajal, Carretera de Colmenar Km 9.1, 28034 Madrid, Spain

N Gallego

C Pérez-Caballero

R Estepa

F Liaño

J Ortuño

Department of Genetics, Universidad Complutense, Madrid, Spain

A Gallego

Correspondence to:

Dr N Gallego

ngallego@hrc.insalud.es

Accepted 4 February 2000 following cardiopulmonary bypass surgery. ${ }^{3}$ In addition the mortality of oliguric neonatal ARF may be as high as $60 \%,{ }^{4}$ and even higher in newborns with congenital heart diseases.

There is a lack of information on ARF from other underlying conditions. We prospectively collected data from 92 consecutive inpatients with ARF but without heart disease, to seek simple clinical parameters that could help paediatric nephrologists establish a reliable prognosis early in the course of ARF. This aspect has not been previously reported to our knowledge. prognosis

\section{Methods}

From 1978 to 1998, a study protocol was applied to 323 consecutive children who fulfilled the criteria of ARF detailed below; 231 patients with congenital heart disease were excluded. The 92 remaining patients were aged 1 day to 18 years (mean 7.35 (SD 5.38) years). There were 48 males and 44 females.

Forms containing clinical details and laboratory investigations of all patients were completed prospectively. Acute renal failure was defined as a sudden increase in serum creatinine (SCr) to more than twice the normal value for the age of the patient, ${ }^{5}$ in patients with previously normal renal function. Oliguria was defined as a urine output less than $1 \mathrm{ml} / \mathrm{kg} / \mathrm{h}$ for more than four hours, resistant to volume repletion, dopamine, and/or frusemide.

Acute renal failure was classified into primary urinary tract related problems (primary UTRP) and non-primary urinary tract related problems (non-primary UTRP). Primary UTRP were nephropathies, uropathies, and toxic causes. Non-primary UTRP were tumour, shock, and others.

When the paediatric nephrologist saw the patient for the first time, clinical conditions evaluated in this study were as follows: hypotension (defined as systolic blood pressure 2 SD below normal values for his/her age and sex in the Spanish paediatric population), ${ }^{6}$ ventilatory support, oliguria, and need for dialysis. Laboratory variables included BUN, $\mathrm{SCr}$, and fractional excretion of sodium.

Complications were analysed and classified into six broad categories: infections, shock, haemorrhage, neurological, respiratory, cardiac, and miscellaneous.

Causes of death were analysed: underlying disease, infections, shock, and cardiac and respiratory failure. In hospital mortality was considered irrespective of whether renal function had been restored before death. Standard dialysis procedures were used to treat hypervolaemia, hyperkalaemia, or acidosis uncontrolled by conservative medical management. Some patients had severe uraemia (BUN above 40 $\mathrm{mmol} / \mathrm{l}$ ) as the main indication for dialysis.

\section{STATISTICAL ANALYSIS}

The variables were studied using a univariate test: the Kruskall-Wallis test for quantitative variables, and the $\chi^{2}$ test with Yates's correction for the qualitative ones. Values are expressed as mean plus or minus SD. A $p$ value of less than 0.05 was considered to indicate significance.

Multiple regression analysis was performed using a stepwise model, considering mortality as the dependent variable, and a set of independent variables, which account for the 
Table 1 Causes and univariate analysis of prognostic risk factors in ARF in children

\begin{tabular}{|c|c|c|c|c|}
\hline & Survivors & Non-survivors & Mortality (\%) & $p$ value \\
\hline All patients & 67 & 25 & 27 & \\
\hline Age (median, years) & 6.6 & 10.3 & & 0.12 \\
\hline \multicolumn{5}{|l|}{ Sex } \\
\hline Males & 36 & 12 & 25 & \\
\hline Females & 31 & 13 & 30 & 0.60 \\
\hline \multicolumn{5}{|l|}{ Primary urinary tract problem } \\
\hline Nephrological & 22 & 0 & 0 & \\
\hline Haemolytic uraemic syndrome & 7 & 0 & 0 & \\
\hline Acute interstitial nephritis & 3 & 0 & 0 & \\
\hline Acute glomerulonephritis & 9 & 0 & 0 & \\
\hline Vasculitis & 2 & 0 & 0 & \\
\hline RVHT + ACEI & 1 & 0 & 0 & \\
\hline Obstructive uropathy & 8 & 0 & 0 & \\
\hline Toxic & 7 & 0 & 0 & \\
\hline Antibiotics & 4 & 0 & 0 & \\
\hline $\mathrm{CuSO}_{4}$ intoxication & 1 & 0 & 0 & \\
\hline Rhabdomyolysis & 2 & 0 & 0 & \\
\hline Total & 37 & 0 & 0 & \\
\hline \multicolumn{5}{|l|}{ Non-primary urinary tract problem } \\
\hline Tumour & 19 & 15 & 44 & 0.98 \\
\hline Bone marrow transplantation & 7 & 10 & 57 & \\
\hline Lymphoma & 2 & 2 & 50 & \\
\hline Osteosarcoma & 6 & 2 & 25 & \\
\hline Leukaemia & 1 & 1 & 50 & \\
\hline CNS tumour & 1 & 1 & 50 & \\
\hline Histiocytosis & 1 & 0 & 0 & \\
\hline Shock & 7 & 8 & 53 & 0.68 \\
\hline Hypovolaemic & 7 & 2 & 22 & \\
\hline Septic & 0 & 3 & 100 & \\
\hline DIC & 0 & 2 & 100 & \\
\hline Lactic acidosis & 1 & 0 & 0 & \\
\hline Others & 4 & 2 & 33 & 0.84 \\
\hline Total & 30 & 25 & 45 & \\
\hline
\end{tabular}

RVHT, renovascular hypertension; ACEI, angiotensin converting enzyme inhibitors; DIC, disseminated intravascular coagulation.

Table 2 Clinical condition of patients when first seen by the nephrologist

\begin{tabular}{|c|c|c|c|c|}
\hline & Survivors & Non-survivors & Mortality (\%) & pvalue \\
\hline Primary urinary tract problem $(n)$ & 37 & 0 & 0 & \\
\hline Hypotension & 2 & - & 0 & \\
\hline Assisted respiration & 2 & - & 0 & \\
\hline Oliguria & 17 & - & 0 & \\
\hline Need for dialysis & 7 & - & 0 & \\
\hline $\mathrm{SCr}(\mu \mathrm{mol} / 1)$ & $3.13(2.79)$ & - & & \\
\hline FENa (\%) & $5.61(3.44)$ & - & & \\
\hline BUN (mmol/l) & $19.3(11.1)$ & - & & \\
\hline Non-primary urinary tract problem $(n)$ & 30 & 25 & 45 & \\
\hline Hypotension & 7 & 15 & 68 & 0.01 \\
\hline Assisted respiration & 4 & 12 & 75 & 0.01 \\
\hline Oliguria & 8 & 10 & 55.5 & 0.45 \\
\hline Need for dialysis & 4 & 15 & 78.9 & 0.00 \\
\hline $\mathrm{SCr}(\mu \mathrm{mol} / 1)$ & $208(118)$ & $256(120)$ & & 0.05 \\
\hline FENa (\%) & $4.97(8.43)$ & $7.5(8)$ & & 0.81 \\
\hline $\mathrm{BUN}(\mathrm{mmol} / \mathrm{l})$ & $15.2(9.1)$ & $28.6(16.7)$ & & 0.00 \\
\hline
\end{tabular}

Values are mean (SD).

FENa, fractional excretion of sodium.

Table 3 Systemic complications in patients with ARF without heart disease

\begin{tabular}{lllll}
\hline & $\begin{array}{l}\text { Survivors } \\
(n=67)\end{array}$ & $\begin{array}{l}\text { Non-survivors } \\
(n=25)\end{array}$ & $\begin{array}{l}\text { Mortality } \\
(\%)\end{array}$ & p value \\
\hline $\begin{array}{l}\text { Primary urinary tract problem } \\
\text { Infections }\end{array}$ & 4 & - & 0 & \\
Cardiac & 1 & - & 0 & \\
Shock & 0 & - & 0 & \\
Respiratory & 1 & - & 0 & \\
Neurological & 3 & - & 0 & \\
Haematological & 2 & - & 0 & \\
Others & 2 & $3.0(1.6)$ & & \\
Non-primary urinary tract problem & 9 & 64.3 & 0.00 \\
Complications per patient & $1.0(1.4)$ & 10 & 71.4 & 0.05 \\
Infections & 5 & 14 & 77.7 & 0.00 \\
Cardiac & 4 & 11 & 78.5 & 0.01 \\
Shock & 4 & 10 & 83 & 0.01 \\
Respiratory & 3 & 17 & 89 & 0.00 \\
Neurological & 2 & 5 & 31 & 0.29 \\
Haematological & 2 & & & \\
Others & 11 & & & \\
\hline
\end{tabular}

patient's clinical data (presence or absence, coded as 1 or 0 ).

\section{Results}

Table 1 shows the causes of ARF and the mortality rates for the entire group and each subgroup. Overall mortality was $27 \%$ (25 cases, 12 males and 13 females; mean age 8.5 (SD 5.9) years, median age 10.3 years, range 1 month to 17 years). There was no difference in mortality between sexes. Age was similar in survivors (median 6.6) and non-survivors $(\mathrm{p}=0.12)$.

Twenty two patients had nephrological disease, obstructive uropathy was present in eight children, and seven had toxic ARF (primary UTRP). No patient in this group died. Thirty four patients had neoplasia, 15 of whom died; shock was present in 15 children, eight of whom died; and six suffered from miscellaneous disorders, two of whom were non-survivors. The mortality rate of this non-primary UTRP group was $45 \%$.

Table 2 shows the clinical situation of each patient when first seen by the nephrologist. In the non-primary UTRP group, hypotension and the need for dialysis and/or mechanical ventilation correlated positively with mortality $(\mathrm{p}<0.01)$. Oliguria was not statistically associated with mortality.

On admission, fractional excretion of sodium was similar in survivor and non-survivor groups. BUN and creatinine values were, however, significantly higher in non-survivors than in the survivor group.

Causes of death were: primary disease in 19 $(22 \%)$, haemorrhage in $19(22 \%)$, infections in $16(19 \%)$, shock in $14(16 \%)$, cardiac failure in seven $(8 \%)$, and respiratory failure in 10 $(12 \%)$. Mean number of causes of death was 1.3 (SD 1.7) per non-surviving patient. Five children died after recovering renal function.

There were 120 complications. Shock, haemorrhage, and the presence of CNS, cardiac, or respiratory complications were predictors of poor outcome $(\mathrm{p}<0.01$; table 3$)$. Each non-survivor developed a mean of three (SD 1.6) complications; in all cases, death resulted from a number of causes. Given the greater number of complications, we did not try to establish a single cause of death.

Stepwise regression analysis shows that the estimation for mortality depends on the following data: presence of hypotension $($ coefficient $=$ 0.278 ), need for ventilation (coefficient = 0.192 ), need for dialysis (coefficient $=0.267$ ), and BUN value (coefficient $=0.012$ ). The $\mathrm{p}$ value of this model was less than $0.0001 ; R^{2}$ was 0.37 .

Stepwise regression analysis shows that the best prediction for mortality can be done using the following score:

Score $=-0.02+0.28$ (hypotension) +0.19 (ventilator) +0.27 (dialysis) $+0.01(\mathrm{BUN})$

Figure 1 shows individual scores for the 55 patients with non-primary UTRP. Note that mortality increases with higher scores. No patient survived if the score value was greater than 0.8 . 


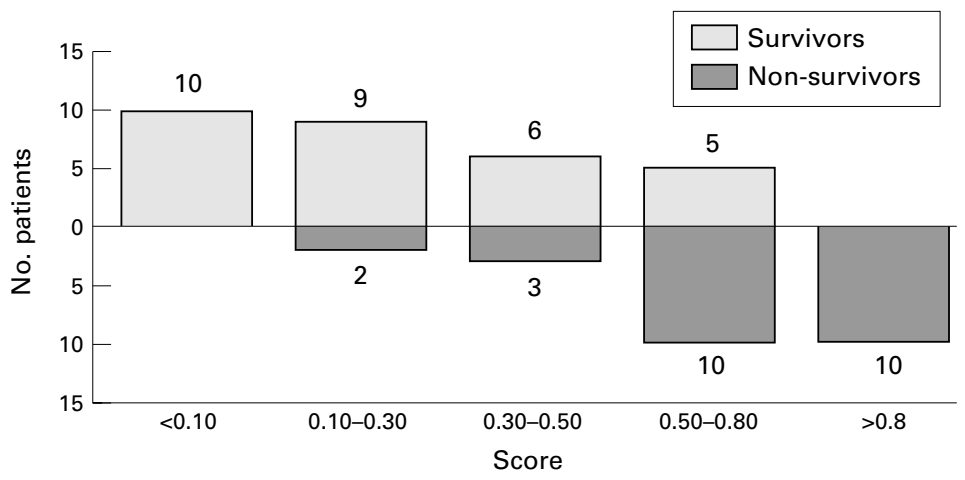

Figure 1 Score of probability of death in 55 patients with ARF and non-primary UTRP, but without heart disease.

\section{Discussion}

In a previous report ${ }^{1}$ we identified prognostic factors in a series of 138 children. In that study most patients $(n=85,62 \%)$ suffered from heart disease, therefore there might be a bias in the results. For this reason we decided to perform a similar study excluding children with cardiopathy. We identified six risk factors in order to establish a prognosis for children who develop ARF not caused by heart disease. Survival rate depended on the underlying disease and on the clinical situation of the patient, as well as the number and characteristics of the complications. Children with non-primary UTRP have a poor outcome. On the contrary, ARF caused by primary UTRP had a good prognosis, as described previously. ${ }^{7}$

Considering the clinical situation of the patient, when first seen by the nephrologist, those needing mechanical ventilation and/or dialysis and those with hypotension and higher values of $\mathrm{SCr}$ and/or BUN had a poor outcome. In addition, mortality was related to the number and extent of complications.

Assisted ventilation and hypotension may reflect a poor haemodynamic situation, and raised SCr and BUN may be a result of poor renal perfusion. Furthermore, death was caused by multiorgan dysfunction syndrome in many non-survivors. In our study, five children died after recovering renal function; ARF was not therefore a contributory cause of death in these cases. Moreover, no patient died because of fluid or electrolyte disturbances. Therefore, it seems that ARF was not the cause of death.

Most children died because of the primary underlying condition, but aetiology was not the only predictive factor. Other factors were: the need for mechanical ventilation, hypotension, SCr and BUN values, as well as the need for dialysis. The need for dialysis depends on the different therapeutic approaches to ARF. Some authors use this technique to treat patients who would not be dialysed by others. Consequently, it is impossible to compare the percentage mortality in dialysed children with other series. Mortality rate is a reflection of the case mix for dialysis.

Barrat and Rigden ${ }^{8}$ dialysed children with ARF following cardiac surgery, if they had oliguria for more than four hours. Others do not consider renal failure treatment in patients with poor prognosis. Our criteria for use of dialysis are described above. All patients in whom dialysis was adequate on a clinical or biochemical basis were dialysed even though they were considered as having a poor prognosis. Published studies on ARF in children rarely try to establish prognostic factors, although Smithies and Cameron $^{9}$ and Stapleton and colleagues ${ }^{4}$ have pointed out the need for this research.

The mortality scores used in intensive care units $^{10}$ are usually applied to predict progress of children with ARF. Many data are needed in order to calculate these scores and not all these variables are determined in patients with ARF. Chevalier et al studied 16 newborn infants with ARF. ${ }^{11}$ In their experience the best prognostic criterion was the duration of oliguria. Postnatal age, birth weight, Apgar score, respiratory status, and BUN and SCr values did not correlate with mortality.

Zobel et al applied several severity scores to 32 patients developing ARF, who were admitted to an intensive care unit and needed dialysis. ${ }^{10}$ A modified APACHE II score established the prognosis better than other scores. However, the usefulness of scores in predicting final prognosis of these children has recently been questioned. ${ }^{2}$ Arora et al analysed 80 children with ARF. ${ }^{3}$ Mortality was related to the underlying disease, anuria, need for dialysis, as well as neurological and respiratory complications. In our study oliguria was not a prognostic factor, but our findings agree with the other conclusions of this report. In our series, we could not evaluate neurological status reliably, mainly because of sedation.

Multivariate regression analysis revealed that the presence of hypotension, high BUN values, and the need for mechanical ventilation and/or dialysis are the only independent risk factors which correlated positively with mortality.

Our results should be verified in a further control population. Other similar studies with more specific pathologies and a higher number of patients are needed to define the prognosis of ARF in childhood.

1 Gallego N, Gallego A, Pascual J, et al. Prognosis of children with acute renal failure: a study of 138 cases. Nephron 1993;64:399-404.

2 Fargason CA, Langman CB. Limitations of the pediatric risk of mortality score in assessing children with acute renal failure. Pediatr Nephrol 1993;7:703-7.

3 Arora P, Kher V, Rai PK, et al. Prognosis of acute renal failure in children: a multivariate analysis. Pediatr Nephrol 1997;11:153-5.

4 Stapleton FB, Jones DP, Gren RS. Acute renal failure in neonates: incidence, etiology and outcome. Pediatr Nephrol 1987:1:314

5 Schwartz GJ, Haycock GB, Edelmann CM, et al. A simple estimate of glomerular filtration rate in children derived estimate of glomerular filtration rate in children derived
from body length and plasma creatinine. Pediatrics

6 Sánchez Bayle M, Estepa R, López Verde L, et al. Valores normales de la tensión arterial en niños españoles. An Esp Pediatr 1984;20:1-7

7 Niaudet P, Haj-Ibrahim M, Gagnadoux MF, et al. Outcome of children with acute renal failure. Kidney Int 1985;28: S148-51.

8 Barrat TM, Ridgen SPA. The kidney following cardiac surgery. In: Gruskin AB, Norman ME, eds. Pediatric nephrology. Developments in nephrology 3. The Hague: Martinus Nijhoff, 1981:278-96.

9 Smithies MN, Cameron JS. Can we predict outcome of acute renal failure? Nephron 1989;51:297-300.

10 Zobel G, Kuttnig M, Ring E, et al. Clinical scoring systems Zobel $G$, Kuttnig M, Ring E, et al. Clinical scoring systems
in children with continuous extracorporeal renal support. Child Nephrol Urol 1990;10:14-17.

11 Chevalier RL, Campbell F, Norman A, et al. Prognostic factors in neonatal acute renal failure. Pediatrics 1984;74:26572 . 